\title{
HOPF CYCLES IN ONE-SECTOR OPTIMAL GROWTH MODELS WITH TIME DELAY
}

\author{
Hitay Özbay and HüSeYin ÇAĞRı SaĞLam \\ Bilkent University \\ Mustafa KeReM YüKSEL \\ University of Turkish Aeronautical Association \\ and \\ Bilkent University
}

This paper analyzes the existence of Hopf bifurcation and establishes the conditions under which the equilibrium path converges toward periodic solutions in a one-sector optimal growth model with delay. We establish the limits and the possibilities of nonlinear dynamics (i.e., cycles) vis-à-vis delays. In particular, we formulate a new method to further comprehend the root distribution of the characteristic equation of a standard optimal growth model with delayed investment structure. We show that nonmonotonic dynamics (limit cycles, persistent oscillations) occurs when the delayed investment causes permanent adjustment failures among the economic variables in the economy.

Keywords: Optimal Growth Models, Time Delay, Hopf Bifurcation

\section{INTRODUCTION}

Cycles have been on the agenda of researchers in economics for at least two centuries. Cycles are shown to be welfare-costly, and thus their stabilization has always been a major political and academic topic. This leads to an evergreen interest of economists in theoretical studies that enlighten the cycle-inducing mechanisms in economic models, as well as empirical studies that test or link firm-level or country-level data with theoretical findings. Their existence, amplitudes, frequencies (or periods), qualities (persistent or decaying cycles), stability, optimality or suboptimality, etc. remain to be fully comprehended.

The main purpose of our paper is to show the existence of Hopf bifurcation and to establish the conditions under which the equilibrium path converges toward the periodic solution in an optimal growth model (OGM) with capital delay. In particular, we contribute to the literature by extending the existing tools in economics to understand and present mechanisms for cycle-inducing investment 
delays and their implications for the macroeconomic dynamics. In other words, we try to establish the limits and possibilities of nonlinear dynamics (i.e., cycles) visà-vis delays. The interesting dynamics (limit cycles, i.e., persistent cycle behavior) occurs when delayed investment causes permanent adjustment failures among the economic variables in the economy.

This literature is often presented as a complementary analysis to real business cycle theory, which states that economic fluctuations are only produced by exogenous stochastic shocks [see Kydland and Prescott (1982); Long and Plosser (1983) for further details]. The real business cycle theory falls short of meeting the necessary shock in terms of the magnitude to match the data. The observed fluctuations, on the other hand, are not regular enough to be explained by periodic cycles. Chaotic solutions are studied to meet this end; however, focus is limited to two-sector models. Hence, to consider cycle-inducing endogenous mechanisms as a complement to the theories resorting to stochastic shocks would enhance the explanatory power of both models.

The question of the effects of delay in economic models has not been exhaustively studied in economic theory. However, the history of such analysis can be roughly separated into two phases, which are determined by the then-current state of economic theory and the elaboration of mathematical tools at hand. Michat Kalecki was the first to introduce a rigorous economic model ${ }^{1}$ and show that business cycles may depend endogenously on production (investment) delays [Kalecki (1935)]. In order to show that cycles are intrinsic to economic behavior, he employs ${ }^{2}$ a linear delay differential equation in the evolution of investment variables. ${ }^{3}$ Kaleckian models exhibit endogenous cycles by employing simple time delays in a linear delay differential equation. Time delays is consistent in the sense that (i) delay structure is empirically significant ${ }^{4}$ and (ii) the first-order linear ordinary differential equations are known to be unable to give cyclic solutions, whereas linear delay differential equations may exhibit endogenous cycles. Zak (1999) employs a production delay in a basic one-sector Solowian model [see Solow (1956)] and, by employing the Hopf bifurcation theorem, shows that the solution path is cyclic under certain conditions, confirming the Kaleckian insight. ${ }^{5}$

The Solow-Kalecki idea has been revived and extended to the Ramsey (1927)type optimal growth model by Asea and Zak (1999), who determine the steady state characteristics of an optimal growth model with capital delay. Asea and Zak (1999) claim that the root distribution of the related characteristic equation contains a pair of pure imaginary eigenvalues and that the model exhibits Hopf cycles. Subsequent research, however, focuses correctly on a system of delay and advance types of differential equations for the first-order conditions for optimal control problems with investment delay and fails to detect analytical cyclic solutions. As Collard et al. (2008) aptly state, "unfortunately, as soon as the dynamics of these models are characterized by a forward looking component, the lack of numerical methods to solve these problems makes the quantitative evaluation of their transitional dynamics difficult."

The complexity of the characteristic equation prevents analytical results, and thus some researchers incline to numerical simulations. Winkler et al. (2004), 
Brandt-Pollmann et al. (2008), and Collard et al. (2008) perform numerical simulations to comprehend the dynamic behavior of optimal growth models with delay. The main findings are summarized by Winkler et al. (2004), who states that "both the frequency and the amplitude of the cycles depend on the length of the investment period," and by Collard et al. (2008), who state that "for a large delay the economy converges to the steady state by oscillations, but consumption smoothing mitigates the induced echo effects through an advanced Euler-type differential." Furthermore, Collard et al. (2006) numerically show that the advanced term in Euler equation governing the dynamic system dampens the fluctuations caused by the delays through a kind of smoothing effect ("time-to-build echo"). Collard et al. (2008) study the short-run dynamics for the convex economy via numerical simulations; Winkler et al. (2004) provide numerical solutions of models of time-delay optimal growth models for a linear limitational production function; Winkler et al. (2005) give a numerical analysis of a time-delayed capital accumulation optimal growth model with a Leontief-type production function; and Brandt-Pollmann et al. (2008) extend the numerical solutions to objective functions with state externalities.

Note that the deficiency of numerical simulations in searching for cyclic solutions is that Hopf bifurcation depends on the precise calibration of the Hopf parameter, and without such calibration it may be impossible to hit the limit cycle solution simply by the randomization of parameters. ${ }^{6}$ Moreover, the quasipolynomial associated with the characteristic equation naturally contains infinitely many complex roots that would result in cyclic behavior. Considering the conditions that exclude completely unstable solutions, such as the transversality condition, it is natural that a random choice of parameters would result in decaying cycles, which is, for the most part, in accordance with the results and interpretation of Collard et al. (2008).

Bambi (2008) exploits the simplified characteristic equation under the AK production function and characterizes a Hopf cycle. In a similar attempt, Winkler (2009) proposes a different approach and associated open-form solutions. Bambi and Gori (2013) introduce indivisible labor supply and Winkler (2011) assumes irreversible investment to the otherwise convex economies ${ }^{7}$ and show that a certain delay parameter causes Hopf cycles. Although these approaches enable analytical results, the main question of whether there exists a limit cycle under a convex economy without any distortions remains unanswered.

In this paper, we formulate a new method for further comprehending the root distribution of the characteristic equation of a standard optimal growth model with delayed investment structure. We extend the analysis of Asea and Zak (1999) and introduce a new technique for the identification of the eigenvalues of the characteristic equation associated with this type of models in a generalized framework. Our method, which employs theoretical as well as numerical techniques, is interdisciplinary in the sense that it exploits substitution of variables to shift and translate the roots of the characteristic equation; Padé approximation to obtain a rational approximation of the exponential time delay component in the characteristic equation; root locus method (control theory) to determine the parameters 
that lead to the pure imaginary eigenvalues of the characteristic equation; grid search (numerical methods) to exactly pin down the parameters; and finally, the Hopf bifurcation theorem (theory of differential equations) to identify the cyclic solution paths.

We show that a one-sector optimal growth model with time-to-build delay admits Hopf cycles, contrary to the conventional results in the literature. Specifically, for a standard optimal growth model with logarithmic utility and Cobb-Douglas production function, the economy is driven into a limit cycle for a sufficiently large production delay. This is in a similar vein to Bambi (2008), where an economically (empirically) questionable but physically viable delay parameter induces cycles. We also reproduce the (numerical) results of Bambi (2008) as a special case.

The paper is organized as follows. To address the effects of delay in reducedform macroeconomic models, we summarize the mathematical instruments in Section 2. Section 3 introduces the model and our approach. Section 4 proposes an algorithm to find the pure imaginary roots for the characteristic equation resulting from optimal growth models with time delay. Finally, Section 5 concludes.

\section{MATHEMATICAL PRELIMINARIES}

The first-order conditions of an economic model with time delay are a (set of) difference-differential equation(s). In Kalecki (1935), this is a one-variable delay differential equation. Inspired by this model per se, Frisch and Holme (1935) first analyze the roots of difference-differential equations of this form. Kalecki is a pioneer in characterizing endogenously driven cycles in the economy as opposed to the cycles determined by exogenous shocks, and his study triggered the development and elaboration of mathematical techniques for the characterization of the stability properties in linear delay differential equations. James and Belz (1938) suggest that "a solution of a difference-differential equation might be developed in terms of an infinite series of characteristic solutions" and investigate "the conditions under which such a development is possible." Hayes (1950) partially closes the literature on roots by giving the properties of the roots of transcendental characteristic equations that frequently occur in dynamic economic systems with delays. ${ }^{8}$ The first thorough analyses of a general class of delay differential equations were by Bellman and Cooke (1963) and Hale (1977).

Early studies are based on the complete root distribution (thus, the resulting dynamics) of the characteristic equation of the model under study given the parameters, whereas contemporary techniques try to determine specific conditions under which specific complex dynamics (such as limit cycles or chaotic behavior) can (or cannot) occur. ${ }^{9}$ Frisch and Holme (1935), James and Belz (1938), and Hayes (1950) are examples of the former approach, whereas Hopf bifurcation ${ }^{10}$ as the main tool for the detection of limit cycles in current studies is an example for the latter. The theorem that characterizes the sufficient conditions under which periodic orbits occur from stationary states is called the Poincaré-Andronov-Hopf theorem.

THEOREM 1 [Poincaré-Andronov-Hopf (Hale and Koçak, 1991, Thm. 11.12, p. 344)]. Let $\dot{\mathbf{x}}=A(\mu) \mathbf{x}+\mathbf{F}(\mu, \mathbf{x})$ be a $C^{k}$, with $k \geq 3$, planar vector field 
depending on a scalar parameter $\mu$ such that $\mathbf{F}(\mu, \mathbf{0})=\mathbf{0}$ and $D_{x} \mathbf{F}(\mu, \mathbf{0})=\mathbf{0}$ for all sufficiently small $|\mu|$. Assume that the linear part $A(\mu)$ at the origin has the eigenvalues $\alpha(\mu) \pm i \beta(\mu)$ with $\alpha(0)=0$ and $\beta(0) \neq 0$. Furthermore, suppose that the eigenvalues cross the imaginary axis with nonzero speed, that is, $\frac{d \alpha}{d \mu}(0) \neq 0$. Then, in any neighborhood $U$ of the origin in $\mathbf{R}^{2}$ and any given $\mu_{0}>0$, there is a $\bar{\mu}$ with $|\bar{\mu}|<\mu_{0}$ such that the differential equation $\dot{\mathbf{x}}=A(\bar{\mu}) \mathbf{x}+\mathbf{F}(\bar{\mu}, \mathbf{x})$ has a nontrivial periodic orbit in $U$.

According to the Hopf theorem, one can summarize the sufficient conditions for Hopf bifurcation as follows:

- (H.1) $A(\mu)$, namely, the Jacobian ${ }^{11}$ of the nonlinear system, has only one pair of pure imaginary eigenvalues (pre-Hopf condition).

- (H.2) Pure imaginary eigenvalues cross the imaginary axis with nonzero speed, i.e., $\frac{d \alpha}{d \mu}(0) \neq 0$ (transverse crossing).

In other words, the roots (eigenvalues) of the Jacobian should lose stability at the critical level of the parameter $\mu$, which is called the Hopf parameter.

As Kind (1999) points out, it is generally easy to prove the existence of Hopf bifurcation because it requires no information on the nonlinear parts of the equation system. Moreover, in systems with dimension higher than two, Hopf bifurcation may be the only tool for the analysis of the cyclical equilibria, ${ }^{12}$ because the Poincaré-Bendixson theorem is not applicable. Furthermore, when the conditions of Hopf bifurcation are satisfied, it guarantees both the existence and the uniqueness of periodic trajectories [Krawiec and Szydłowski (1999)]. However, the Hopf theorem gives no information on the number or the stability of closed orbits. On the other hand, nonlinear parts can be used for the calculation of a stability coefficient in order to determine the stability properties of the closed orbits [Kind (1999)]. Guckenheimer and Holmes (1983, Thm 3.4.2, pp. 151-153) provide both the theory and an example in that direction.

\section{HOPF CYCLES IN OPTIMAL GROWTH MODELS WITH TIME DELAY}

Consider an economy that is inhabited by infinitely lived households with unit aggregate measure. The representative individual's preferences are represented by a twice continuously differentiable, strictly increasing, and strictly concave utility function $u(c)$ and a subjective discount rate $r>0$. In this economy, it takes $\tau \geq 0$ periods to install new capital equipment. The production function $f(k)$ is strictly concave and twice continuously diffferentiable. We assume that the utility function and the production function satisfy Inada conditions. The infinite horizon planning problem is given by

$$
\begin{array}{ll}
\max _{\{c(t)\}_{t=0}^{\infty}} & \int_{0}^{\infty} e^{-r t} u(c(t)) d t \\
\text { subject to } & \\
& k(t)=f(k(t-\tau))-\delta k(t-\tau)-c(t), \\
& k(t)=\phi(t), t \in[-\tau, 0],
\end{array}
$$


where $0<c(t)<f(k(t-\tau)), \delta \in[0,1]$ is the capital depreciation rate, $k(t-\tau)$ is the productive capital stock at time $t$, and $\phi(t)$ is the initial capital function. We employed a modified version of the Maximum Principle [see Kolmanovskii and Myshkis (1992)] to identify the first-order necessary conditions:

$$
\begin{aligned}
& \dot{c}(t)=r \frac{u^{\prime}(c(t))}{u^{\prime \prime}(c(t))}-e^{-r \tau} \frac{u^{\prime}(c(t+\tau))}{u^{\prime \prime}(c(t))}\left[f^{\prime}(k(t))-\delta\right], \\
& \dot{k}(t)=f(k(t-\tau))-\delta k(t-\tau)-c(t) .
\end{aligned}
$$

Note that the first-order conditions have delayed terms as well as advanced terms. The optimizing household takes the investment delay into account when considering consuming today or tomorrow. ${ }^{13}$ In other words, the marginal productivity of capital available at time $t+\tau$ is weighted by the ratio of the marginal utility of consumption at $t+\tau$ to the marginal utility of consumption at $t$ [see also Collard et al. (2008, p. 132)]. Also note that, as $\tau \rightarrow 0$, the first-order conditions converge to those of the standard neoclassical growth model, and as $\alpha \rightarrow 1$, the production schedule becomes AK, and the first-order conditions are the same as those of Bambi (2008). The steady state equations are

$$
r=e^{-r \tau}\left(f^{\prime}\left(k_{\mathrm{ss}}\right)-\delta\right) \quad \text { and } \quad c_{\mathrm{ss}}=f\left(k_{\mathrm{ss}}\right)-\delta k_{\mathrm{ss}} .
$$

Assuming that $f$ is strictly concave, there is a negative relation between the timeto-build delay and the steady state level of capital. This is because the lower the delay is, the higher is the effective capital used in production. The characteristic equation associated with the first-order conditions (2) and (3) around the steady state is

$$
h(\lambda ; \Lambda) \equiv\left(r-r e^{\lambda \tau}-\lambda\right)\left(r e^{r \tau} e^{-\lambda \tau}-\lambda\right)-e^{-r \tau} \Lambda^{2}=0,
$$

where $\Lambda^{2}=\left.\frac{u^{\prime}(c)}{u^{\prime \prime}(c)} f^{\prime \prime}(k)\right|_{c_{s s}, k_{s s}} \in \mathbf{R}$. Under the standard assumptions of convex preferences and convace production, we have ${ }^{14} \Lambda^{2} \geq 0$.

As $h(\lambda ; \Lambda)$ is a quasi-polynomial, it can be generalized as

$$
H(\lambda):=A(\lambda)+B(\lambda) e^{\lambda \tau}+C(\lambda) e^{-\lambda \tau},
$$

where $A(),. B($.$) , and C($.$) are real coefficient polynomials of various degrees$ themselves. Note that the roots of a quasi-polynomial, and thus of the equation (4), come in complex conjugate pairs.

PROPOSITION 1. Solutions $\lambda$ to the equation (4) are symmetric with respect to $\operatorname{Re} \lambda=\frac{r}{2}$. In other words, $\lambda$ is a solution to the equation (4) if and only if $r-\lambda$ is a solution.

Proof. Define $\lambda^{*}=r-\lambda$. Then

$$
h(\lambda ; \Lambda)=h(r-\lambda ; \Lambda)=h\left(\lambda^{*} ; \Lambda\right)
$$

concludes the proof. 
Proposition (1) is crucial for various aspects. The symmetry implies that an optimal growth model with time delay problem is governed by the infinite number of stable and unstable complex eigenvalues. This already explains the decaying cycle solutions for the numerical simulations so far presented. With the appropriate parameter and initial condition adjustments, the unstable solutions are suppressed and stable decaying cyclic solutions remain.

Recall that the strategy for finding Hopf cycles is to find a pure imaginary pair of eigenvalues. Suppose such a pair $\pm j \omega$ exists. Then, by symmetry, $r \mp j \omega$ is also a solution. Proposition (1) verifies that the eigenvalues $\{ \pm j \omega, r \mp j \omega\}$ are symmetric around $\operatorname{Re} \lambda=\frac{r}{2}$. Defining $\bar{\lambda}=\lambda+\frac{r}{2}$, the roots of the equation (4) are shifted to the left by $\operatorname{Re} \lambda=\frac{r}{2}$. In other words, the roots of the shifted characteristic equation,

$$
h(\bar{\lambda} ; \Lambda) \equiv\left(\frac{r}{2}-r e^{\frac{r \tau}{2}} e^{\bar{\lambda} \tau}-\bar{\lambda}\right)\left(r e^{\frac{r \tau}{2}} e^{-\bar{\lambda} \tau}-\bar{\lambda}-\frac{r}{2}\right)-e^{-r \tau} \Lambda^{2}=0,
$$

are symmetric with respect to the imaginary axis and equation (4) has a pair of pure imaginary roots if and only if equation (5) has a pair of complex roots with $\operatorname{Re} \bar{\lambda}= \pm \frac{r}{2}$. Further define

$$
\begin{aligned}
\sigma & =\frac{r \tau}{2}, \\
s & =\bar{\lambda} \tau, \\
\gamma & =\Lambda \tau,
\end{aligned}
$$

so that every parameter is scaled with respect to $\tau$. Then, after substitution and multiplication of (5) by -1 , we obtain

$$
g(s ; \sigma, \gamma) \equiv\left(\sigma+s-2 \sigma e^{\sigma-s}\right)\left(\sigma-s-2 \sigma e^{\sigma+s}\right)+\gamma^{2} e^{-2 \sigma}=0 .
$$

Note that equation (5) has a pair of complex roots with $\operatorname{Re} \bar{\lambda}= \pm \frac{r}{2}$ if and only if equation (6) has a pair of complex roots with $\operatorname{Re} s= \pm \sigma$. Define

$$
G(s)=\left(\frac{\sigma-s}{\sigma+s-2 \sigma e^{\sigma-s}}\right)\left(\frac{e^{-\sigma}}{\sigma+s}\right) .
$$

Then $g(s ; \sigma, \gamma)=0$ can be restated as

$$
1+\gamma^{2} G(s) G(-s)=0 .
$$

In control theory, $G(s)$ can be seen as the transfer function of a retarded time delay system [Michiels and Niculescu (2007)]. A transfer function $G(s)$ is further called proper if it satisfies $\lim _{|s| \rightarrow \infty}|G(s)|<\infty$, and strictly proper if $\lim _{|s| \rightarrow \infty}|G(s)|=$ 0 . If $G^{-1}(s)$ is also proper, then $G(s)$ is called bi-proper. $G(s)$ is called stable if complex poles reside in the open left half of the complex plane, that is, it has no poles in $\overline{\mathbf{C}}_{+}$. Note that the first component of $G(s)$, namely $\left(\frac{\sigma-s}{\sigma+s-2 \sigma e^{\sigma-s}}\right)$, is 
stable $^{15}$ and bi-proper; and the second component of $G(s),\left(\frac{e^{-\sigma}}{\sigma+s}\right)$, is stable and strictly proper.

\section{ROOTS}

In what follows, we employ numerical methods to evaluate the existence of complex conjugate roots of equation (7). Simply, we will follow the following algorithm: We approximate the exponential terms using Padé approximation. Varying $\gamma^{2}$, we trace the solutions in the complex plane using the root locus method. We identify the roots that cross $\operatorname{Re} s= \pm \sigma$. We then fine tune the precision errors due to Padé approximation and use grid search to sort out the exact value of $\gamma^{2}$ that admits roots with $\operatorname{Re} s= \pm \sigma$. Then we discuss the supportability of such values with respect to economic parameters.

The solutions to the equation (7) can be analyzed by employing the root locus method well known in control theory. Root locus "primarily deals with finding the roots of a characteristic polynomial that is an affine function of a single parameter, say $K$,

$$
\chi(s)=D(s)+K N(s),
$$

where $D(s)$ and $N(s)$ are fixed monic polynomials" [Özbay (2000, pp. 64-65)]. We can restate the problem as

$$
1+K G^{*}(s)=0
$$

by defining $G^{*}(s)=\frac{N(s)}{D(s)}$ [Özbay (2000, p. 66)]. In other words, the root locus technique can be used to "examine how closed-loop system poles change as $K$ varies from 0 to $+\infty$, or from 0 to $-\infty$ " [Özbay (2000, p. 66)]. Equation (7) is exactly equation (8) when $G^{*}(s)=G(s) G(-s)$ and $K=\gamma^{2}$. Thus, if a pair of complex conjugate roots (or more) cross(es) the vertical line $\operatorname{Re} s= \pm \sigma$ as $K$ $\left(=\gamma^{2}\right)$ varies, it can be ensured that equation (7) has a pair of complex conjugate roots (or more) with $\operatorname{Re} s= \pm \sigma$ for some value of $K\left(=\gamma^{2}\right)$, because there is a continuous relation between the roots of equation (7) and the coefficient $K$.

For a given value of $\sigma, G^{*}(s)$ contains exponential terms. These terms appear because of the time delay in the model. Padé approximation is employed for a rational approximation of the time delay component. The exponential term $e^{-\tau s}$ can be Padé approximated as

$$
e^{-\tau s} \approx P_{d}(s)=\frac{\sum_{k=0}^{n}(-1)^{k} \vartheta_{k} \tau^{k} s^{k}}{\sum_{k=0}^{n} \vartheta_{k} \tau^{k} s^{k}},
$$

where the coefficients $\vartheta_{k}$ are

$$
\vartheta_{k}=\frac{(2 n-k) ! n !}{2 n ! k !(n-k) !}, \quad k=0,1, \ldots, n .
$$




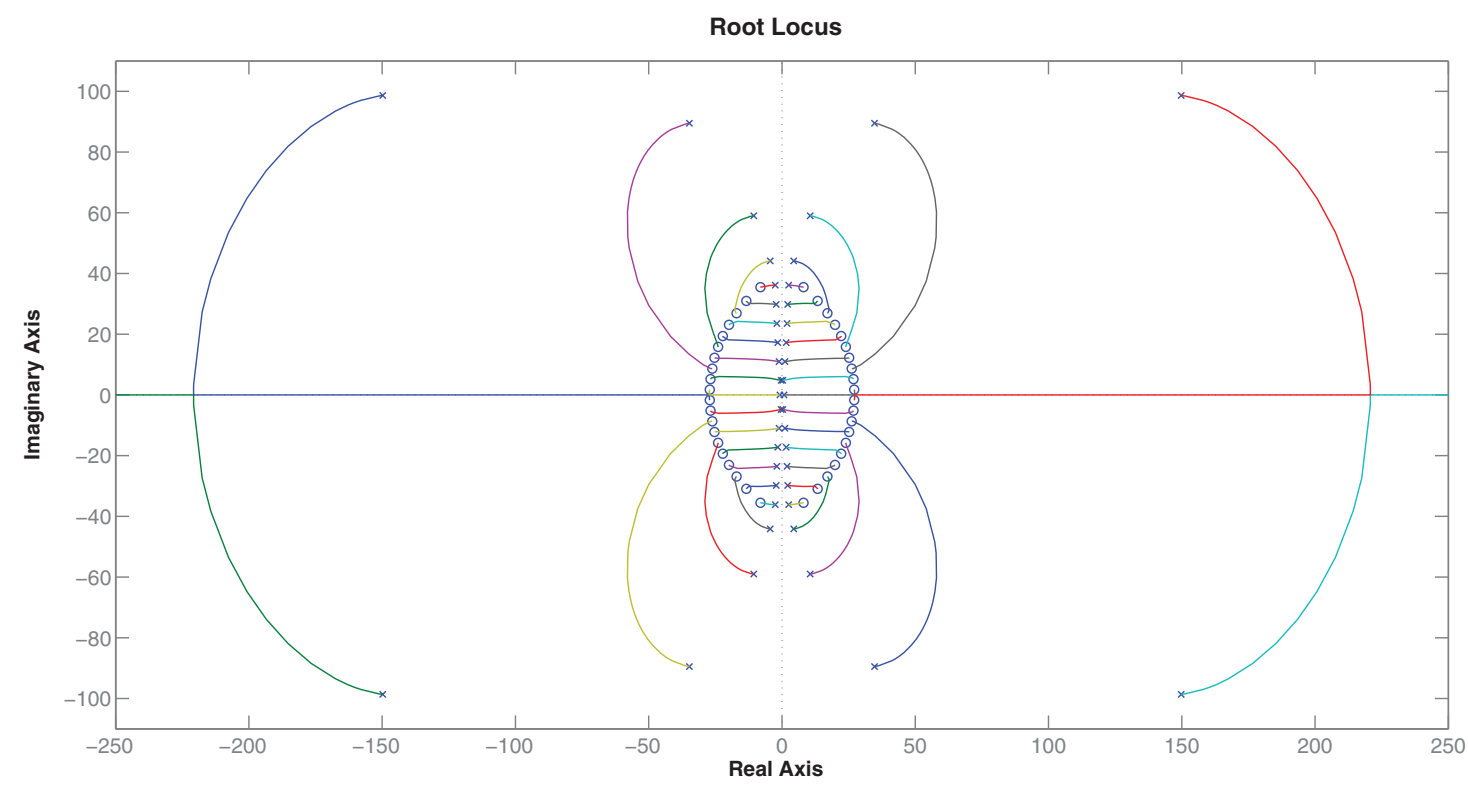

Figure 1. Root locus for $1+\gamma^{2} G(s) G(-s)=0$ when $\sigma=0.8$.

The degree of approximation, $n=20$, is assumed to be sufficiently high for the numerical analysis carried out in the paper ${ }^{16}$ [for Padé approximation, as well as its approximation errors, subsequent discussions and examples, see Özbay (2000, Sect. 7)].

We temporarily set $\sigma=0.8$ for illustrative purposes. Figure 1 presents the movement of the roots of equation (7) in the complex plane as $K\left(=\gamma^{2}\right)$ changes. When $K\left(=\gamma^{2}\right)$ is close to zero, the roots are given by a cross sign, and as $K$ $\left(=\gamma^{2}\right)$ goes to infinity, they move toward the ones given with zero sign.

We are specifically interested in whether there exists a root with $\operatorname{Re} s= \pm \sigma$. Figure 2 focuses on the roots around $\operatorname{Re} s= \pm \sigma= \pm 0.8$.

It is obvious from Figure 2 that a symmetric couple of roots cross the line $\operatorname{Re} s= \pm \sigma$. According to the analysis carried out so far, when $\sigma=0.8$, equation (7) admits roots at $s= \pm 0.8 \pm 4.88 j$ when $\gamma^{2}=56.8$. A grid search around these values confirms that when

$$
\gamma^{2}=56.413919919919920,
$$

the value of $1+\gamma^{2} G(s) G(-s)$ at

$$
s= \pm 0.8 \pm 4.867024361111118 j
$$

is close to zero, with an error of $6.9094 \times 10^{-6}$ in absolute value. ${ }^{17}$ Rearranging the characteristic equation (4) in terms of $\sigma$ and $\gamma^{2}$, we obtain

$$
h\left(\sigma, \lambda ; \gamma^{2}\right) \equiv\left(2 \sigma-2 \sigma e^{2 \sigma}-\lambda \tau\right)\left(2 \sigma e 2^{\sigma} e^{-\lambda \tau}-\lambda \tau\right)-\gamma^{2} e^{-2 \sigma}=0 .
$$

For a given $\gamma^{2}$, the values attained by $h\left(\sigma, \lambda ; \gamma^{2}\right)$ in absolute value for different values of $\sigma$ and $\lambda=j \omega$ are plotted in Figure 3. The $\sigma$ and $\lambda=j \omega$ values at which $h\left(\sigma, \lambda ; \gamma^{2}\right)$ attains its minimum give the corresponding $\sigma$ at which the pure 


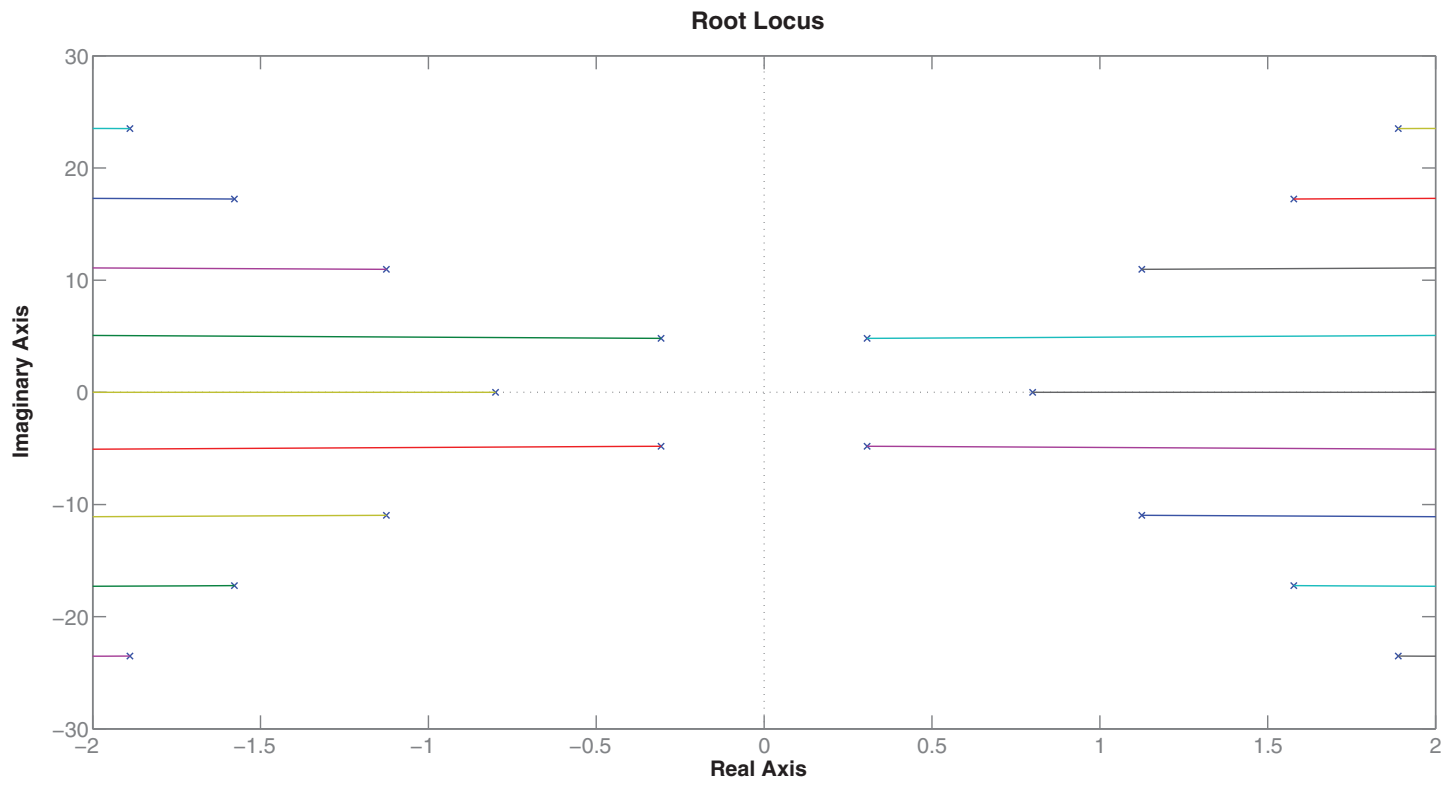

FigURE 2. Root locus for $1+\gamma^{2} G(s) G(-s)=0$ when $\sigma=0.8[-2<\operatorname{Re} s<2]$.

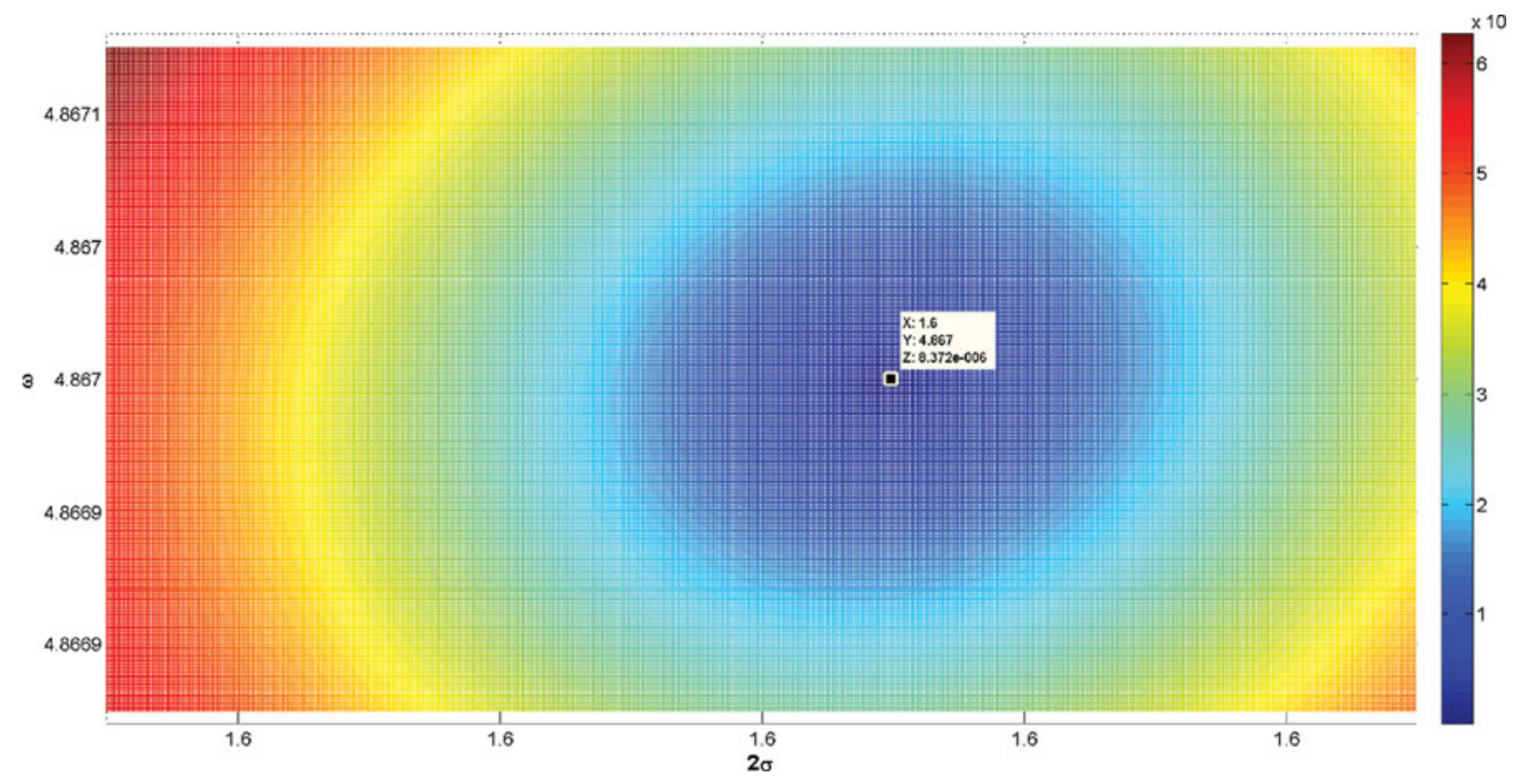

FiguRE 3. $h\left(\sigma, \lambda ; \gamma^{2}\right)$ in absolute value for different values of $\sigma$ and $\lambda=j \omega\left[\gamma^{2}=\right.$ 56.413919919919920]. The minimizing $\sigma$ and $\lambda=j \omega$ pair is shown on the graph.

imaginary eigenvalue occurs and the corresponding pure imaginary eigenvalue. The minimizing $\sigma(=0.8)$ and $\lambda=j \omega(=4.86702425 j)$ pair is shown on the graph. Point $Z$ on the graph is the value of $h\left(\sigma, \lambda ; \gamma^{2}\right)$ and is $3.701645 \times 10^{-6}$. In other words, for the given combination of $\left(\sigma, \lambda ; \gamma^{2}\right)$, the characteristic equation $h\left(\sigma, \lambda ; \gamma^{2}\right)$ is close to zero, with an error on the order of $10^{-6}$ 's.

At this point, we have to go back to the original economy to see whether there exist plausible parameters that support the Hopf cycle inducing $\gamma^{2}$. Recall that $\gamma^{2}=\left.\tau^{2} \frac{u^{\prime}(c)}{u^{\prime \prime}(c)} f^{\prime \prime}(k)\right|_{c_{s s}, k_{s s}}$. Thus, $\gamma^{2}$ depends on the parameters of the economy as well as the time-to-build delay. ${ }^{18}$ In Example 4, we give numerical values of 
a convex economy that exhibits Hopf cycles, ${ }^{19}$ in which the parameters of the economy are physically plausible (whereas economically questionable).

\section{Example 1}

Assume logarithmic utility and Cobb-Douglas production technology with capital elasticity $\alpha$, i.e., $f(k)=A k^{\alpha}$. Consider the following parameterization:

$$
r=0.05, \delta=0.05, \tau=32 \text {. }
$$

Then, for $\alpha_{\text {Hopf }}=0.59156291 \approx 0.6$, equation $(4)$ has a pair of pure imaginary roots such that

$$
\lambda= \pm \frac{4.867024361111118}{32} j= \pm 0.152094511285 j
$$

Also note that the period $T$ of the Hopf cycle is $\frac{2 \pi}{\omega} \tau=1.29097 \tau(=41.311)$.

It is important to note that given any $\pm \sigma \in \mathbf{R}$, the existence of complex conjugate roots $s$ with $\operatorname{Re} s= \pm \sigma$, i.e., $s= \pm \sigma \pm j \omega$, is independent of the form of the utility and production functions as long as these functions are twice continuously differentiable and their second derivatives are both negative. In other words, the analysis that leads to Example 4 can be separated into two distinct parts: (i) given any $\pm \sigma= \pm \frac{r \tau}{2} \in \mathbf{R}$, find a $\bar{\gamma}^{2}$ such that there exist a pair of complex conjugate roots $s$ with $\operatorname{Re} s= \pm \sigma$; and (ii) calibrate the parameters of the economy so that $\gamma^{2}(r, \delta, \tau, \alpha)$ meets $\bar{\gamma}^{2}$. Proposition 2 summarizes the numerical results that constrain $\pm \sigma= \pm \frac{r \tau}{2} \in \mathbf{R}$, allowing a pair of complex conjugate roots $s$ with $\operatorname{Re} s= \pm \sigma$.

PROPOSITION 2. For every $\sigma=\frac{r \tau}{2} \in(0.646564819216,0.903122510938)$ there exists a pair of complex conjugate roots $s$ with $\operatorname{Re} s= \pm \sigma$, i.e., $s= \pm \sigma \pm j \omega$, with $\left( \pm \omega, \bar{\gamma}^{2}\right) \approx( \pm 4.71238898038,0)$ at the lower bound and $\left( \pm \omega, \bar{\gamma}^{2}\right)=$ $( \pm 4.94346026026,133.032532533)$ at the upper bound.

One important result is that the results presented in Proposition 2 match those of Bambi (2008, pp. 1,038-1,039). As $\bar{\gamma}^{2}$ tends to 0, we should have the capital elasticity of production technology $\alpha$ tending to 1 so that the production function should converge to an AK technology. Adopting AK technology and letting $\sigma=$ $W\left(\frac{3 \pi}{2}\right) / 2=0.646564818939690$, where $W(s)$ is a Lambert function, Bambi (2008) characterizes the Hopf-inducing pure imaginary root as $\omega= \pm \frac{3 \pi}{2} j=$ $\pm 4.712388980384690 j$. Note that Bambi (2008) and Proposition 2reciprocally verify each other. Moreover, the $2 \pi$ periodicity of the Lambert function implies that we can further characterize other solutions for $\bar{\gamma}^{2}=0$. In Figure 4 we plot the first 20 solutions for $\omega=2(n-1) \pi \pm \frac{3 \pi}{2} j$ as $n=1,2, \ldots, 20$.

Note that the first solution indicates that the Hopf-inducing $(\sigma, \omega) \approx$ $(0.6466,4.712)$ couple is in accordance with the preceding findings. 


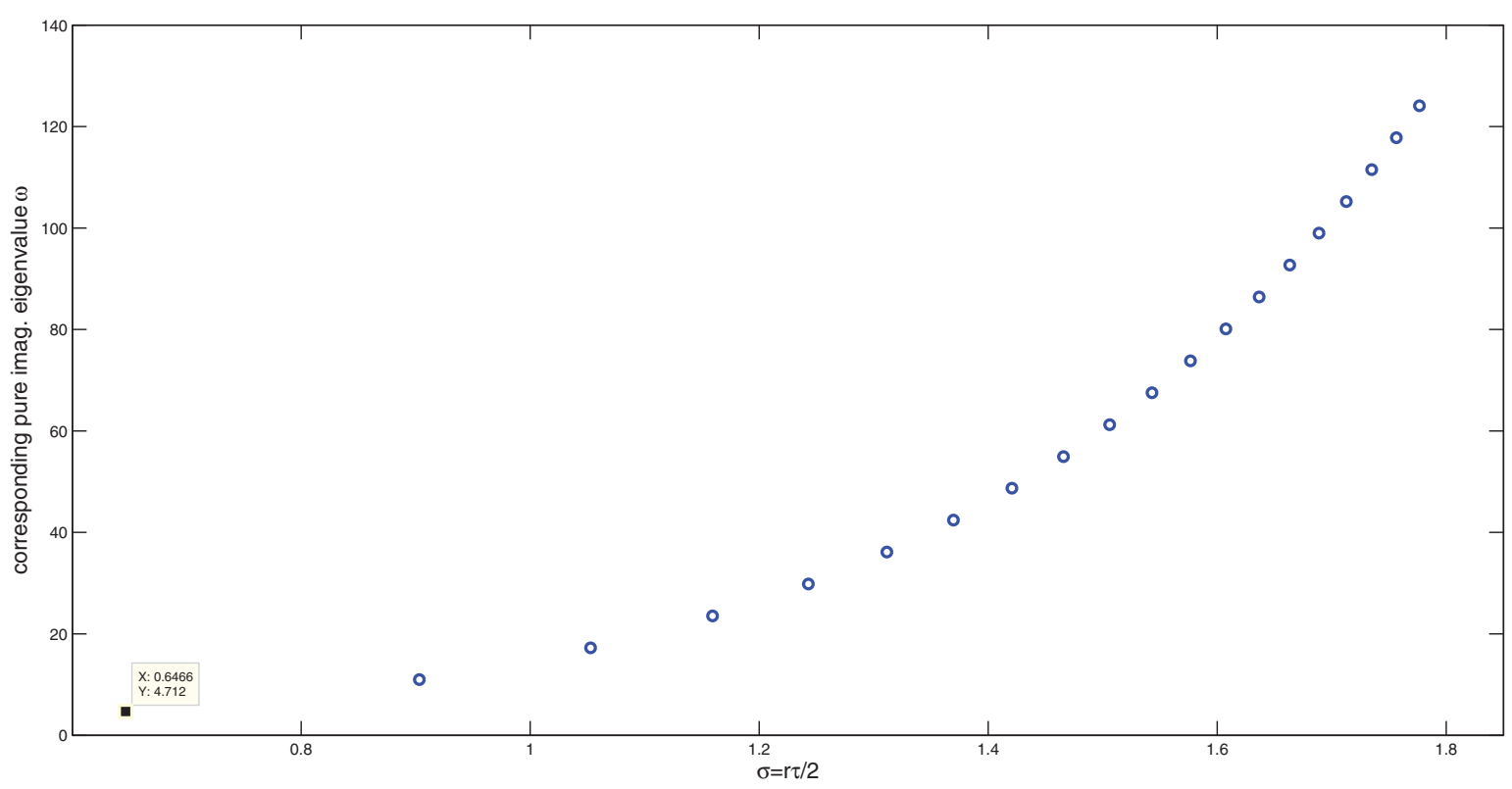

FIGURE 4. Pure imaginary solutions to the characteristic equation for different parameter combinations when $\bar{\gamma}^{2}=0$, i.e., AK production.

\section{CONCLUSION}

In this paper we formulate a new method to further comprehend the root distribution of the characteristic equation of a standard optimal growth model with delayed investment structure. We conclude that a one-sector convex optimal growth model with time-to-build lag admits Hopf cycles, contrary to the conventional results in the literature. The Hopf-inducing delay parameters are economically (empirically) questionable but physically viable.

\section{NOTES}

1. A brief exposition of the Kalecki (1935) model and its properties can be found in Zak (1999) and Szydłowski (2002). These texts reproduce Kalecki's results with contemporary techniques, which are also employed in this paper.

2. Michał Kalecki studied the underlying forces of cycles in economy throughout his life, and his theories vary from linear difference differential equation systems to exogenous factors. As Besomi (2006) pointed out, in his study of Kalecki's business cycle theories, Kalecki "either failed to provide a rigorous proof of the stability of the cycle when the model was endogenous or failed to provide an explanation of the cycle relying on the properties of the economic system, resorting instead to exogenous shocks to explain the persistence of fluctuations." Kalecki interpreted cycles as the dynamic expression of the "intrinsic antagonism of capitalism"; however, he "acknowledged the existence of disturbing factors, from which he abstracted in order to isolate a pure cycle." Besomi (2006) also reports that "Kalecki's models describes damped fluctuations around a line of stationary equilibrium and rely for the persistence of fluctuations on exogenous shocks" and, moreover, all his models "crucially depend for cyclicality upon one or more reaction lags."

3. The exact linear delay differential equation studied by Kalecki $\left(1935\right.$, p. 332) is $\dot{J}(t)=\frac{m}{\theta} J(t)-$ $\frac{m+n \theta}{\theta} J(t-\theta)$, where $m$ and $n$ are constant.

4. Kalecki (1935, pp. 337-338) estimates the lag between the curves of beginning and termination of building schemes (dwelling, industrial, and public buildings) as 8 months and lags between orders and deliveries in the machinery-making industry as 6 months, based on data supplied by the German 
Institut fuer Konjunkturforschung. He assumes that "the average duration of $\theta$ is 0.6 years" [Kalecki (1935)].

5. Yüksel (2011) introduces a matrix method to identify the pure imaginary roots in a SolowianKaleckian economy with endogenous population growth.

6. Kaldor (1940) also criticizes Kalecki (1935) by pointing out that the drawback of the model is that "the existence of an undamped cycle can be shown only as a result of a happy coincidence, of a particular constellation of the various time-lags and parameters assumed" and "the amplitude of the cycle depends on the size of the initial shock."

7. By the term "convex," we mean the standard neoclassical assumptions of convex preferences and concave production.

8. The particular form of equations in Hayes (1950) is $\tau(s)=s e^{s}-a_{1} e^{s}-a_{2}=0$. This type of transcendental equations are called quasi-polynomials.

9. See among others, Medio (1998), Barnett et al. (2015), and Barnett and Guo (2015) for detailed surveys on nonlinear and complex dynamics and bifurcations in economic models.

10. "In 1942, Hopf published the ground-breaking work in which he presented the conditions necessary for the appearance of periodic solutions, represented in phase space by a limit cycle" [Szydłowski (2002)]. The original article is in German [see Hopf (1942)]. An English translation, with comments, is included as Section 5 in Marsden and McCracken (1976).

11. Note that this is nothing but the Jacobian matrix that results from linearization of the system, if the system is nonlinear. If $\overline{\mathbf{x}}$ is the equilibrium point of $\dot{\mathbf{x}}=\mathbf{f}(\mathbf{x})$, i.e., $\mathbf{f}(\overline{\mathbf{x}})=\mathbf{0}$, then the linear differential equation $\dot{\mathbf{x}}=D \mathbf{f}(\overline{\mathbf{x}}) \mathbf{x}=\left.\left(\frac{\partial f_{i}}{\partial x_{j}}(\overline{\mathbf{x}})\right)\right|_{i, j} \mathbf{x}$ is the linear variational equation or the linearization of the vector field $\mathbf{f}$ at the equilibrium point $\overline{\mathbf{x}}$ [Hale and Koçak (1991, Def. 9.4, p. 267)].

12. Asea and Zak (1999, p. 1164, footnote 13) mention other ways in which periodic orbits may arise. Heteroclinic orbits are given as an option, yet these are stated to be "rare."

13. The first-order conditions in Asea and Zak (1999) are only delayed type because "the co-state variable has the same timing convention as the time the decision is made" (p. 1166). In other words, the consumer in Asea and Zak (1999) does not take the future shadow price of capital into consideration. In that respect, our consumer has "deeper" perfect foresight [see also Bambi (2008, footnote 2)].

14. The square notation is used for practical consistency and will be clear in the sections to come.

15. It can be easily shown that the only root of $\left(\sigma+s-2 \sigma e^{\sigma-s}\right)$ in $\mathbf{C}_{+}$is at $s=\sigma$, which is canceled by the numerator polynomial.

16. In fact the error due to Padé approximation is not of primary concern, because we further our analysis beyond the results of the Padé approximation using a grid search method to find the exact root with $\operatorname{Re} s= \pm \sigma$ and the exact value of $\gamma^{2}$ that allows for this root. Padé approximation is only employed to narrow down the area of the complex plane that should be grid-searched.

17. The degree of error is within acceptable limits considering the precision errors of the numerical computing environment due to rounding.

18. For an economy with logarithmic utility $[u(c)=\ln c]$ and Cobb-Douglas production technology with capital elasticity $\alpha$, i.e., $f(k)=A k^{\alpha}$, we have

$$
\gamma^{2}=\frac{(1-\alpha)}{\alpha} \tau^{2}\left(\delta+r e^{r \tau}\right)\left((1-\alpha) \delta+r e^{r \tau}\right) .
$$

The analysis starts with the choice of $\sigma$. By rearranging the terms, we obtain

$$
\gamma^{2}=\frac{(1-\alpha)}{\alpha}\left(\delta \tau+\sigma e^{\sigma}\right)\left((1-\alpha) \delta \tau+\sigma e^{\sigma}\right) .
$$

Thus, $\gamma^{2}$ is only a function of $\alpha, \delta$ and $\tau\left(=\frac{2 \sigma}{r}\right)$.

19. Hopf bifurcation analysis boils down to finding a pair of pure imaginary roots, sincebecause the nonzero speed condition is not actually necessary. To be more specific: "[the nonzero speed condition] is expressed by saying that the pair of complex conjugate eigenvalues crosses the imaginary axis with nonzero speed. This is also a generic requirement, though it is not absolutely necessary: the existence part of the theorem remains valid even in the degenerate case when this derivative is zero [etc.]" [see Farkas (1994, p. 418)]. 


\section{REFERENCES}

Asea, Patrick K. and Paul J. Zak (1999) Time-to-build and cycles. Journal of Economic Dynamics and Control 23, 1155-1175.

Bambi, Mauro (2008) Endogenous growth and time-to-build: The AK case. Journal of Economic Dynamics and Control 32, 1015-1040.

Bambi, Mauro and Franco Gori (2013) Unifying time-to-build theory. Macroeconomic Dynamics 18, 1713-1725.

Barnett, William A. and Guo Chen (2015) Bifurcation of macroeconometric models and robustness of dynamical inferences. Foundations and Trends in Econometrics 8(1-2), 1-144.

Barnett, William A., Apostolos Serletis, and Demitre Serletis (2015) Nonlinear and complex dynamics in economics. Macroeconomic Dynamics 19, 1749-1779.

Bellman, Richard E. and Cooke, Kenneth L. (1963) Differential-Delay Equations. New York: Academic Press.

Besomi, Daniele (2006) Formal modelling vs. insight in Kalecki's theory of the business cycle. Research in the History of Economic Thought and Methodology 24, 1-48.

Brandt-Pollmann, Ulrich, Ralph Winkler, Sebastian Sager, Ulf Moslener, and Johannes P. Schlöder (2008) Numerical solution of optimal control problems with constant control delays. Computational Economics 31, 181-206.

Collard, Fabrice, Omar Licandro, and Luis A. Puch (2006) Time-to-Build Echoes. Working paper 16, Fundación de Estudios de Economía Aplicada (FEDEA).

Collard, Fabrice, Omar Licandro, and Luis A. Puch (2008) The short-run dynamics of optimal growth models with delays. Annales d'Economie et de Statistique 90, 127-143.

Farkas, Miklós (1994) Periodic Motions. New York: Springer-Verlag.

Frisch, Ragnar and Harold Holme (1935) The characteristic solutions of a mixed difference and differential equation occuring in economic dynamics. Econometrica 3, 225-239.

Guckenheimer, John and Philip Holmes (1983) Nonlinear Oscillations, Dynamical Systems and Bifurcations of Vector Fields. New York: Springer-Verlag.

Hale, Jack (1977) Theory of Functional Differential Equations. New York: Springer-Verlag.

Hale, Jack and Hüseyin Koçak (1991) Dynamics and Bifurcations. New York: Springer-Verlag.

Hayes, N.D. (1950) Roots of the transcendental equation associated with a certain differencedifferential equation. Journal of the London Mathematical Society 25, 226-232.

Hopf, Eberhard (1942) Abzweigung einer periodischen Lösung von einer stationären Lösung eines Differentialsystems. Berichten der Mathematisch-Physischen Klasse der Sächsischen Akademie der Wissenschaften zu Leipzig 94, 1-22.

James, R.W. and M.H. Belz (1938) The significance of the characteristic solutions of mixed difference and differential equations. Econometrica 6, 326-343.

Kaldor, Nicholas (1940) A model of the trade cycle. Economic Journal 50, 78-92.

Kalecki, Michał (1935) A macrodynamic theory of the business cycle. Econometrica 3, 327-344.

Kind, Christoph (1999) Remarks on the economic interpretation of Hopf bifurcations. Economic Letters 62, 147-154.

Kolmanovskii, Vladimir Borisovich and Anatolii Dmitrievich Myshkis (1992) Applied Theory of Functional Differential Equations. Dordrecht, Netherlands: Academic Publishers.

Krawiec, Adam and Marek Szydłowski (1999) The Kaldor-Kalecki business cycle model. Annals of Operational Research 89, 89-100.

Kydland, Finn E. and Edward C. Prescott (1982) Time to build and aggregate fluctuations. Econometrica 50(6), 1345-1370.

Long, John B., Jr. and Charles I. Plosser (1983) Real business cycles. Journal of Political Economy 91(1), 39-69.

Marsden, Jerrold E. and Marjorie McCracken (1976) The Hopf Bifurcation and Its Applications. New York: Springer-Verlag.

Medio, Alfredo (1998) Nonlinear dynamics and chaos: I. A geometrical approach. Macroeconomic Dynamics 2, 505-532. 
Michiels, Wim and Silviu-Iulian Niculescu (2007) Stability and Stabilization of Time Delay Systems: An Eigenvalue-based Approach. Philadelphia: SIAM.

Özbay, Hitay (2000) Introduction to Feedback Control Theory. Boca Raton, FL: CRC Press LLC.

Ramsey, F.P. (1927) A contribution to the theory of taxation. Economic Journal 37(145), 47-61.

Solow, Robert (1956) A contribution to the theory of economic growth. Quarterly Journal of Economics 70, 65-94.

Szydłowski, Marek (2002) Time-to-build in dynamics of economic Models: I. Kalecki's model. Chaos, Solitons and Fractals 14, 697-703.

Winkler, Ralph (2009) Time-to-Build in the AK Growth Model: Short-Run Dynamics and Response to Exogenous Shocks. Mimeo, CER-ETH, Center for Economic Research at Eidgenössische Technische Hochschule (ETH) Zurich.

Winkler, Ralph (2011) A note on the optimal control stocks accumulating with a delay. Macroeconomic Dynamics 15, 565-578.

Winkler, Ralph, Ulrich Brandt-Pollmann, Ulf Moslener, and Johannes P. Schlöder (2004) Time lags in capital accumulation. In D. Ahr, R. Fahrion, M. Oswald, and G. Reinelt (eds.), Operations Research Proceedings 2003, pp. 451-458. Heidelberg, Germany: Springer.

Winkler, Ralph, Ulrich Brandt-Pollmann, Ulf Moslener, and Johannes P. Schlöder (2005) On the Transition from Instantaneous to Time-Lagged Capital Accumulation: The Case of LeontiefType Production Functions. Discussion paper 05-30, ZEW-Zentrum für Europäische Wirtschaftsforschung/Center for European Economic Research.

Yüksel, Mustafa Kerem (2011) Capital dependent population growth induces cycles. Chaos, Solitons \& Fractals 44(9), 759-763.

Zak, Paul (1999) Kaleckian lags in general equilibrium. Review of Political Economy 11, 321-330. 